\title{
Radio Emission from the Sun and Stars: New Insights into Energetic Phenomena
}

\section{T. S. Bastian}

National Radio Astronomy Observatory, Charlottesville, VA 22903 USA

\begin{abstract}
Energetic phenomena on the Sun and late-type stars pose a number of fascinating puzzles. These include coronal heating, flares, and coronal mass ejections, all believed to be manifestations of magnetic energy release. Radio radiation is a sensitive tracer of energetic phenomena on both the Sun and stars. Radio observations of the Sun over the past decade have produced new insights into the physics of magnetic energy release in flares and coronal mass ejections. Radio observations of latetype stars have exploited sensitive imaging and spectroscopic techniques to further constrain the nature of the relevant emission mechanisms. A surprise has been the recent discovery of radio emission from brown dwarf stars, implying the existence of substantial magnetic fields and a means of dissipating magnetic energy, neither of which are understood.
\end{abstract}

\section{Introduction}

Radio emission has been detected from stars and stellar systems across the HR diagram (Fig. 1). A striking feature of the radio HR diagram is the preponderance of nonthermal radio emitters from the middle through the lower right of the diagram, which includes the RS Canum Venaticorum (RS CVn) and Algoltype close binaries, pre-main-sequence weak-lined $\mathrm{T}$ Tauri stars, main sequence dwarfs, and the recent and surprising detections of brown dwarfs. A common feature of these objects is substantial convective envelopes of the constituent stars - or in the case of brown dwarfs, substellar objects - presumed to play a critical role in the creation and evolution of magnetic fields. Nonthermal radio emission is a tracer of energetic electrons that are accelerated in physical processes that dissipate magnetic energy. Hence, studies of nonthermal radio emission on stars yield insights into stellar magnetic fields and magnetic energy release.

Solar radiophysics has provided a critical reference for stellar radio emission, providing an interpretive framework for stellar phenomena which appear to be analogous to those on the Sun - e.g. flares - although care must be taken to avoid leaning on solar/stellar analogies too heavily. The radio emitting properties of the Sun and stars have been reviewed extensively. Aspects of solar radio emission are covered by White \& Kundu (1997), Bastian, Benz, \& Gary (1998), and the volume edited by Gary \& Keller (2004). Numerous reviews of the radio emitting properties of stars track the progress of the past decade (e.g., Seaquist 1993, Güdel 1994, André 1996, Bastian 1996, van den Oord 1996, White 1996, 


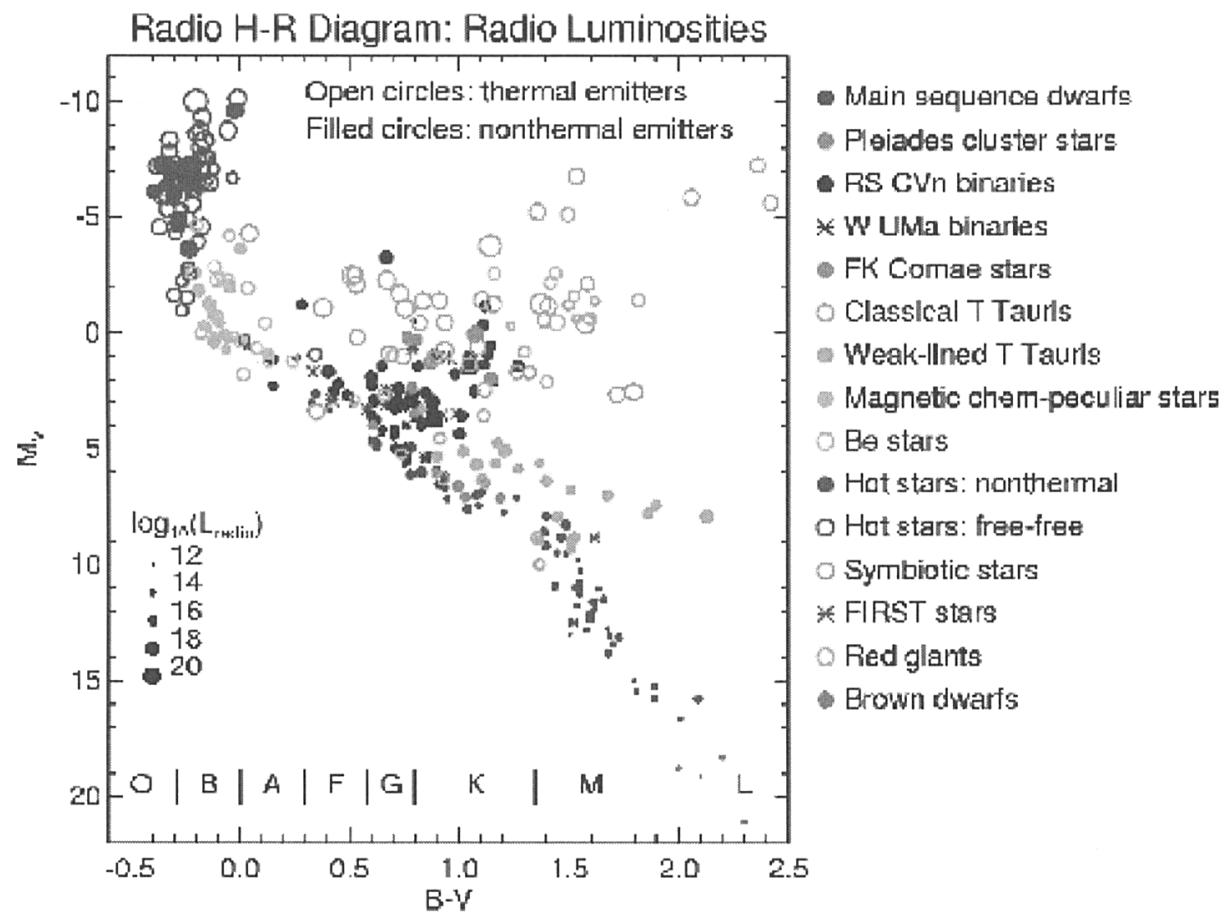

Figure 1. Radio emission across the HR diagram. The symbol size corresponds to the logarithm of the radio luminosity. Open symbols represent thermal radio emitters and solid symbols represent nonthermal radio emitters.

2000), the most recent being the excellent review by Güdel (2002). This being the case, the present review is topical in nature, drawing attention to several developments over the past decade in radio studies of both solar and stellar radio emission related to magnetic energy release.

This review is organized as follows: in $\S 2$, radio emission mechanisms relevant to the Sun and stars are briefly discussed. In $\S 3$, certain results regarding radio emission from solar flares and CMEs are summarized. In $\S 4 \mathrm{I}$ turn to recent observational work on active stars, notably RS CVn binaries, flare stars (dMe stars), and brown dwarfs. The opportunities presented by the next generation of radio instrumentation are highlighted in $\S 5$ and I conclude in $\S 6$.

\section{Radio emission mechanisms}

Two "natural" frequencies of the outer atmosphere of the Sun and stars - the electron cyclotron frequency $\left(\nu_{B e}=e B / 2 \pi m_{e} c=2.8 B \mathrm{MHz}\right)$ and the electron plasma frequency $\left(\nu_{p e}=\sqrt{n_{e} e^{2} / \pi m_{e}}=9 n_{e}^{1 / 2} \mathrm{kHz}\right)$ - fall in the radio regime. Consequently, the gyromagnetic and plasma radiation mechanisms play a prominent role in solar and stellar radio emission. 
Plasma radiation results from the nonlinear conversion of Langmuir waves to electromagnetic waves at the electron plasma frequency $\nu_{p e}$ or its harmonic $2 \nu_{p e}$. Langmuir waves, in turn, are excited by a number of nonequilibrium electron distributions; an electron beam or a loss-cone distribution, for example. Plasma radiation is relevant to solar radio bursts at frequencies $<1-2 \mathrm{GHz}$, including the classical solar radio bursts at meter wavelengths; i.e., solar radio bursts of type I, II, III, IV, and V (although gyrosynchrotron radiation likely plays a role in certain type IV radio bursts). Type II radio bursts are believed to be the result of coronal shocks. As the shock propagates super-Alfvénically to greater coronal heights, the associated plasma radiation slowly drifts to lower frequencies. Type III radio bursts are excited by the propagation of electron beams in the corona. As the electron beams propagate at $\sim 0.1-0.3 c$, fast-drift radio bursts result. Type II radio bursts are often associated with CMEs while type III radio bursts are commonly associated with impulsive flares. Both can accompany a given event, however.

Gyromagnetic emission results from the spiraling electron motion in magnetic fields. A strong magnetic field, typically $200-2000 \mathrm{G}$ on the Sun, is sufficient to render the ambient coronal optically thick to thermal gyroresonance absorption at low harmonics of the electron gyrofrequency $\nu_{B} e$ (see White \& Kundu 1997 for a review). Thermal gyroresonance emission is relevant to solar active regions. In flares, electrons are heated to a high temperature $\left(T \sim 2 \times 10^{7} \mathrm{~K}\right)$ and/or accelerated to energies of $10 \mathrm{~s}$ of $\mathrm{keV}$ to several Mev, or more. These electrons emit thermal and/or nonthermal gyrosynchrotron radiation in a broadband continuum which dominates the radio emission from in solar flares at frequencies $z 2-3 \mathrm{GHz}$. The emission typically shows a spectral maximum at $5-10 \mathrm{GHz}$ although it can be much higher. As shown in $\S 3.2$, nonthermal gyrosynchrotron radiation also plays a role in radio CMEs at meter and decimeter wavelengths, or frequencies of $\approx 100-500 \mathrm{MHz}$.

Thermal free-free radiation, due to collisions between free electrons and ions in the atmosphere of the Sun or a stars, is ubiquitous but does not play a significant role in energetic phenomena. Additional mechanisms such as the cyclotron maser (Melrose \& Dulk 1982; Melnikov \& Fleishman 1999) or transition radiation (Fleishman \& Kahler 1992) may play significant roles under some circumstances, but this remains to be demonstrated.

\section{The Sun}

Significant progress in understanding energetic phenomena on the Sun, notably flares and coronal mass ejections (CMEs), has been made during the course of the past decade. Critical to this progress has been the availablility of observations across the electromagnetic spectrum. Hence, soft-X-ray (SXR) and hard-X-ray (HXR) imaging observations from Yohkoh, HXR spectroscopy from CGRO/BATSE, UV/EUV imaging and spectroscopy from SOHO, coronagraphic observations from $\mathrm{SOHO}$, and optical observations from the ground, have all played important roles. At radio wavelengths, the Very Large Array (VLA), the Nobeyama Radioheliograph (NoRH), the Owens Valley Solar Array (OVSA), and the Nançay Radioheliograph (NRH) have all played important roles. A comprehensive review of radio emission from energetic processes on 
the Sun is beyond the scope of this review. The reader is referred to the review by Bastian et al. (1998). Here we draw attention to a small number of developments regarding flares and CMEs.

\subsection{Solar flares}

Progress in understanding radio emission from flares has been made in two areas in recent years: 1) broadband digital spectroscopy at decimeter and meter wavelengths $(\sim 100 \mathrm{MHz}$ to a few $\mathrm{GHz})$ and their relation to magnetic energy release, and 2) high-time-resolution imaging of solar flares at centimeter wavelengths and the nature of flaring coronal loops.

Beginning in the 1980s, type-III-like radio bursts (now referred to as type IIIdm bursts) were recognized at frequencies $>300 \mathrm{MHz}$ (Elgaroy 1980; Benz et al. 1981), differing from classical type III radio bursts to the extent that they occurred at higher frequencies and that they showed both positive (reverse-slope) and negative drift rates corresponding to electron beams that were propagating downward or upward in the solar corona. Isliker \& Benz (1994) showed that the most common coherent burst type between $1-3 \mathrm{GHz}$ were reverse-slope type IIIdm bursts. Subsequent studies of the correlation between type IIIdm bursts and HXR bursts showed that, unlike classical type III radio bursts at meter wavelengths, for which there is only a 3correlation with HXR bursts, reverse-slope type IIIdm bursts were correlated 48of sequences of type IIIdm bursts and HXR data led Aschwanden et al. (1995a,b) to conclude that there is a causal relation between upward-directed electron beams and type III bursts and downwarddirected electron beams and HXR pulses during impulsive energy release in flares. The implication is that magnetic energy release accelerates multitudes of electron beams that produce the type IIIdm bursts. The downward-direct electron beams are correlated with HXR events produced by thick-target emission from more energetic electrons. The missing ingredient here is imaging observations of type IIIdm bursts which may provide a new diagnostic of the energy release location and process.

At centimeter wavelengths, the NoRH in Japan provided dedicated imaging of the Sun at $17 \mathrm{GHz}$ starting in 1992 with support of $34 \mathrm{GHz}$ added in 1996 (Nakajima et al. 1993). With an angular resolution comparable to the SXR and HXR imagers on board the Yohkoh spacecraft, the NoRH allowed the relationship between radio emissions and X-ray emissions to be elucidated in both the spatial and temporal domains. Important contributions have also been made by the OVSA. An analysis by Wang et al. (1995) used Yohkoh and OVSA observations of an impulsive flare to show that the radio footpoints were connected by a SXR loop, that the dominant HXR source was associated with the more weakly magnetized footpoint, while the dominant radio source was associated with the more strongly magnetized footpoint. These observations confirmed the basic picture wherein energetic electrons are injected into a coronal magnetic loop and emit nonthermal gyrosynchrotron radiation. Electrons with small pitch angles precipitate from the loop and are stopped on the cold chromosphere where they emit HXRs via nonthermal bremsstrahlung. Electrons with sufficiently large pitch angles remain trapped in the magnetic loop until Coulomb collisions or wave/particle interactions cause them, too, to precipitate from the loop. An 
asymmetric loop causes enhanced precipitation at one footpoint and enhanced gyrosynchrotron at the conjugate footpoint.

Detailed morphological studies of HXR and radio emission from impulsive flares were carried out by Hanaoka $(1996,1997)$ and by Nishio et al. (1997) who found that double-loop configurations played a role in the majority of flares, arising from the emergence of new magnetic flux emerging into a pre-existing active region.

\subsection{Coronal mass ejections}

It is generally accepted that type II radio bursts are tracers of fast MHD shocks. Although $60-80 \%$ of all type II radio bursts are associated with a CME, the causes of coronal type II radio bursts remain somewhat controversial. Probable causes include a coronal blast wave initiated, perhaps, by a solar flare (Uchida 1974, Wagner \& MacQueen 1983; Cane \& Reames 1988); fast mass ejecta (Gopalswamy et al. 1997); or by a CME (Cliver et al. 1999, Classen \& Aurass 2002). Hence type II radio bursts are not necessarily reliable tracers of CMEs and, in any case, suitable instrumentation does not currently exist to iamge complete type II radiative signatures.

Bastian \& Gary (1997) have considered incoherent thermal and nonthermal radio signatures of CMEs. Thermal radio emission was apparently detected from a CME near the plasma level by Gopalswamy \& Kundu (1993) at 38, 50, and $74 \mathrm{MHz}$, and has been detected more recently by Kathaviran, Ramesh, \& Subramanian (2002) and by Ramesh, Kathaviran, \& Sastry (2003) at 109 $\mathrm{MHz}$ using the Gauribindanur Radioheliograph. Bastian et al. (2001) used the Nançay Radioheliograph to image nonthermal gyrosynchrotron radiation from a $\mathrm{CME}$ at five discrete frequencies between $150-450 \mathrm{MHz}$. The emission was due to $\mathrm{MeV}$ electrons entrained in the magnetic field of the CME. They found that the multi-point radio spectra allowed them to impose strong constraints on the magnetic field strength and on the density of the thermal plasma in the CME. Hence, incoherent radiative signatures of CMEs at radio wavelengths now allow additional diagnostics to be brought to bear on the problem of understanding the CME phenomenon.

A fascinating and important topic that is related to both flares and CMEs is that of solar energetic particle events and the identification and interpretation of radio signatures associated with SEP events (e.g., shock-associated type III radio bursts, shock-accelerated type III bursts, type III-l bursts). This topic, however, is well beyond the scope of this review.

\section{Late type stars and substellar objects}

Progress in understanding radio emission from late-type stars has proceeded at a slower pace than is the case for the Sun, but there has nevertheless been progress along several lines. Only a few can be highlighted here: imaging observations of active binaries and flares stars, spectroscopy of flare stars, and radio detections of brown dwarfs. 


\subsection{RS CVn binaries}

The RS Canum Venaticorum (RS CVn) type active binaries have been studied for more than two decades. They are short period $\left(\mathrm{P}_{\text {orb }} \sim 1-14\right.$ days $)$ binary systems, typically composed of a dwarf star and a subgiant. They display greatly enhanced levels of activity (Hall 1976; Fekel, Moffet, \& Henry 1986): strong chromospheric emission, star spots, soft X-ray emission, and nonthermal radio emission. Many RS CVn systems produce large flares which manifest themselves in the optical, UV, X-ray, and radio bands. These flares can release a total energy of $10^{34}-1-^{36}$ ergs; that is, $10^{2}-10^{4}$ times that released in large solar flares.

The essential features of both quiescent and flaring emission at centimeter wavelengths from RS-CVn-type binaries were established in the mid-1980s using VLBI techniques. Mutel et al. (1985) showed that the quiescent component was typically characterized by emission on the scale of the binary system and a brightness temperature $T_{B} \sim 10^{9} \mathrm{~K}$. The emission is characterized by a flat spectrum between $1-15 \mathrm{GHz}$ and is moderately circularly polarized. Flaring sources are substantially more compact, with angular sizes comparable to the radius of the active star, inverted spectra, a low degree of circular polarization, and a high brightness $\left(T_{B} \gtrsim 10^{10} \mathrm{~K}\right)$. Occasionally, both components - a "corehalo" morphology are seen.

Since the work of Mutel et al., which relied on fitting VLBI visibility measurements obtained from heterogeneous arrays of radio telescopes, the Very Long Baseline Array (VLBA) has become available, a homogeneous array of ten radio telescopes that has largely standardized VLBI techniques and has allowed sensitive new measurements to be made. These observations (e.g., Lestrade et al. 1999; Beasley \& Güdel 2002; Trigilio et al. 2002; Ransom et al. 2002) largely confirm the earlier results. An outstanding example of the VLBA's sensitivity is the observation reported by Beasley \& Güdel (2000), who mapped UX Ari in quiescence at $8.4 \mathrm{GHz}$. The source was comparable to the scale of the binary but the peak flux was $1.5 \mathrm{mJy} /$ beam, corresponding to a brightness temperature of only $T_{B} \sim 2.6 \times 10^{7} \mathrm{~K}$, agreeing, perhaps fortuitously, with contemporaneous observations of the thermal x-ray-emitting plasma observed by ASCA . A large scale gradient in the degree of circular polarization was also seen, suggesting that the magnetic field was organized on the scale of the system. Attempts to account for the observed radio emission in terms of thermal, rather than nonthermal, gyrosynchrotron radiation failed. Multiband VLA and VLBA observations of AR Lac by Trigilio et al. (2001), plus contemporaneous X-ray (Beppo SAX) observations, were used to conclude that the radio emission could not result from the same distribution function of electrons as those responsible for the X-ray emission; that nonthermal gyrosynchrotron radiation was responsible although the thermal X-ray and nonthermal radio emission could perhaps be cospatial.

White \& Franciosini (1995) addressed the radio spectrum of RS CVn binaries. It had been previously established that quiescent radio emission was circularly polarized and that the sense of polarization often reversed between 1.5 and $5 \mathrm{GHz}$ (Mutel et al. 1987), interpreted as a result of the nonthermal gyrosynchrotron emission becoming self absorbed. Monitoring observations of several RS CVn binaries with the VLA in the $1.5 \mathrm{GHz}$ by White \& Franciosini convincingly established that the polarization reversal is due to coherent plasma radiation playing an increasing role $\lesssim 3 \mathrm{GHz}$. Hence, as is the case for the 
(flaring) Sun, incoherent nonthermal gyrosynchrotron appears to satisfactorily account for radio emission from the quiescent corona of $\mathrm{RS} \mathrm{CVn}$ binaries but coherent plasma radiation plays a role below $\sim 3 \mathrm{GHz}$.

\subsection{Imaging and spectroscopy of flare stars}

Progress in understanding radio emission from flare stars has proceeded along two lines in recent years. First, like the active binaries, dMe flare stars have attracted the attention of the VLBI community. It has been suspected for many years that, like the active binaries, quiescent radio emission from dMe coronae is due to nonthermal gyrosynchrotron radiation from a sustained population of mildly relativistic electrons although here, too, it is suspected that coherent processes play a role at low frequencies. VLBI techniques were brought to bear on flare stars beginning with observations of YZ CMi by Benz \& Alef (1991). Subsequent observations by Benz, Alef, \& Güdel (1995), Benz et al. (1998), and most recently by Petalozzi et al. (2000) have constrained the size of the radio corona on these objects. While the radio corona on flare stars appears to be considerably more extended than is the case for the Sun, it is still sufficiently compact to be consistent with the hypothesis that nonthermal gyrosynchrotron radiation is indeed the relevant emission mechanism.

Beginning with Bastian \& Bookbinder (1987), spectroscopic observations of coherent radio bursts at low frequencies $(\$ 1-2 \mathrm{GHz})$ have strived to constrain the their basic properties in order to identify the relevant emission mechanism(s). Observations were obtained by the Arecibo telescope (Bastian et al. 1990, Abada-Simon et al. 1994, 1997), the Bonn telescope (Stepanov et al. 1995, 2001), or combinations of telescopes (Güdel et al. 1989). While these observations have provided tantalizing clues as to the nature of the mechanisms at work, they were frustrated by a lack of bandwidth. Observations were typically limited to $\Delta \nu / \nu$ of a few percent.

With the completion of the Arecibo upgrade, this has now changed. Two critical elements of the upgrade - the construction of a ground screen and the addition of a Gregorian optical system - allow sensitive broadband observations to be carried out, with $\Delta \nu / \nu$ of $\sim 50 \%$. The bandwidth is now sufficient that the identification of stellar burst types and their mechanisms is a realistic expectation. To this end, Osten \& Bastian (2003) performed pilot observations of AD Leo using the Wideband Arecibo Pulsar Processor (WAPP). They observed $\mathrm{AD}$ Leo with a time resolution of $10 \mathrm{msec}$ over a frequency range of $1.1-1.6 \mathrm{GHz}$ and detected several bursts. A preliminary analysis has revealed fast drift bursts highly reminiscent of solar type-III-like bursts.

\subsection{Radio detections of brown dwarfs}

Following the discovery of the first brown dwarf, Gl 229B, by Nakajima et al. (1995) the study of brown dwarfs has blossomed into a new observational discipline (see review by Basri 2000). A number of important puzzles have been posed by the observations regarding magnetic activity at the bottom of the main sequence. On the one hand, with the marked decline of $\mathrm{H} \alpha$ emission for spectral types later than M9, and the ubiquity of rapid rotation, the activity-rotation relation that holds for solar-type stars appears to break down (Mohanty \& Basri 2003). On the other hand, with the detection of a soft X-ray flare on the M9 
brown dwarf LP994-20 by Rutledge et al. (2000) followed by the detection of a flare and quiescent emission on the same object at radio wavelengths by Berger et al. (2001), vigorous magnetic activity nevertheless occurs. The review by Basri (2004) provides a more comprehensive treatment of this important problem and we touch on the radio observations here.

Since Berger's detection of LP994-20 at radio wavelengths, 12 late M and L dwarfs have been observed by the VLA, 3 of which were detected both in flaring and quiescent states (Berger 2002), including an L3.5 object (2MASS 0036+18). The level of quiescent emission is of order $100 \mu \mathrm{Jy}$ and that of the flares is $\sim 1$ mJy on all sources. The flaring component appears to be significantly circularly polarized. All three objects detected at radio wavelengths are rapid rotators $\left(v \sin i>30 \mathrm{~km} \mathrm{~s}^{-1}\right)$ whereas the nondetections for which rotational velocities were available ( 3 objects) all had $v \sin i<10 \mathrm{~km} \mathrm{~s}^{-1}$, perhaps indicating that rapid rotators are more likely to be radio emitters, although this clearly requires much more work.

A striking feature of the radio emission detected from objects at the bottom of the main sequence is that they appear to violate the correlation between thermal SXR and nonthermal radio emission established by Benz \& Güdel (1994) for solar-type stars: these objects appear to be grossly overluminous in their radio emission, adding another puzzle to the nature of magnetic activity at the bottom of the main sequence. Further progress will require positive identification of the radio emission mechanism(s) responsible for the quiescent and flaring emissions. A coherent mechanism could possibly account for the anomalously high radio luminosity and the relatively high degree of circular polarization but the observations are not sufficiently well-constrained to make this determination. Alternatively, fundamentally new, non-solar-like models of the radio source may be necessary. Given that these objects have largely neutral atmospheres, yet are magnetized and are rapid rotators, planetary magnetospheric models may have more relevance here than solar-type models.

\section{A look forward: future instrumentation}

The coming decade promises dramatic progress in understanding the physics of the Sun and stars as a result of new observations made possible by the next generation of radio instrumentation. Four instruments are briefly described here that will have a profound impact on solar and stellar radiophysics and the contribution radio observations will make to a more general understanding of the Sun and stars.

\subsection{The Frequency Agile Solar Radiotelescope}

FASR is a solar dedicated radio telescope designed to perform broadband imaging spectroscopy over nearly three decades of bandwidth (Bastian 2002). Like other modern radio telescopes, FASR will employ arrays of antennas to perform Fourier synthesis imaging. Unlike other radio telescopes, however, FASR will be designed to image the Sun's radio emission from $\sim 0.1-30 \mathrm{GHz}$ on a time scale and with a frequency resolution commensurate with the phenomenon under study. In the case of flares, for example, FASR will sample the entire frequency range on a time scale of $100 \mathrm{msec}$ with a spectral resolution of $1 \%$. 
FASR will therefore allow radio diagnostics of physical processes in the Sun's outer atmosphere - from the middle chromosphere into the upper corona - to be fully exploited for the first time.

The key science goals of the FASR projects are discussed in detail elsewhere (see Gary \& Keller 2004). Briefly, FASR will open the field of solar coronal magnetography, where a variety of techniques will be exploited to make quantitative measurements of the coronal magnetic field. In addition, FASR is expected to make unique contributions to the physics of flares, coronal mass ejections, and the quiet Sun. A unique feature of the instrument is that it provide an integrated picture of solar phenomena from chromospheric to coronal heights. Many of the lessons learned from FASR will convey to radio emission from solar-like stars.

\subsection{The Expanded Very Large Array}

The NRAO has embarked on a major upgrade of the VLA. Current plans call for two phases to the upgrade, the first of which is underway. The first phase will produce three major new capabilities: 1) rather than being confined to observations in several discrete, narrow frequency bands, the EVLA will be able to observe at any frequency between 1-50 GHz and in up to four independently tunable pairs of frequencies in a given bands; 2) the continuum sensitivity of the array will be improved by factors of a few $(\nu<10 \mathrm{GHz})$ to more than a factor of $20(\nu=10-50 \mathrm{GHz}) ; 3)$ a new correlator will provide many more frequency channels (at least 16384), process up to $8 \mathrm{GHz}$ of bandwidth in each polarization channel, and provide vastly higher spectral resolution. The second phase of the upgrade will 1) increase the angular resolution of the instrument by an order of magnitude by adding eight new antennas on long baselines; 2) add frequency coverage from $0.24-1.2 \mathrm{GHz}$ through the addition of UHF packages at the prime focus; 3 ) add a new ultra-compact antenna configuration designed to improve the instrument's sensitivity to low-surface-brightness emission.

The EVLA will revitalize the field of stellar radio astronomy by greatly improving the statistics of known classes of radio emitters, discovering new classes of radio emitters and, largely through the leverage provided by the full frequency coverage from $0.24-50 \mathrm{GHz}$ and its superior sensitivity, allow new radio diagnostics to be be exploited. Particular exciting are prospects for progress in understanding stellar magnetic fields and stellar magnetic activity.

\subsection{The Atacama Large Millimeter Array}

ALMA is a joint project of the NRAO and ESO to build a Fourier synthesis telescope operating between $36-850 \mathrm{GHz}$, or 350 microns to $10 \mathrm{~mm}$. ALMA will be composed of $64,12 \mathrm{~m}$ antennas, located in the Chajnantor region of the Atacama desert in Chile at an elevation of $5000 \mathrm{~m}$. Like the VLA/EVLA, the ALMA antennas will be reconfigurable. The highest angular resolution that it will achieve is $\theta=0.02 \lambda_{m m}^{\prime \prime}$. ALMA is being designed to address an extremely wide range of astrophysics, including the formation of galaxies and clusters, star formation, planet formation, the creation of the elements, and low temperature thermal science such as planetary composition, the structure of interstellar gas and dust, astrochemistry, and the origins of life. ALMA will also excel at detecting chromospheric emission from solar-like stars, thereby providing a critical observational link between optical and UV observations of photospheric 
and chromospheric emission lines, and X-ray and radio observations of stellar coronae.

\subsection{The Low Frequency Array}

LOFAR will address the long-neglected low frequency range from $20-240 \mathrm{MHz}$, corresponding to wavelengths of $1.25-15 \mathrm{~m}$ (e.g., Kassim et al. 2002). Previously, low frequency observations were strongly limited by the influence of the Earth's ionosphere and the angular resolution of imaging observations were consequently limited to arcminutes. These limitations have now been overcome (e.g., Kassim et al. 1993) and high resolution imaging observations of sidereal sources are now possible (although imaging in the solar system will still be limited by scattering in the corona and the interplanetary medium).

Planning is underway to enable LOFAR to perform solar observations (White, Kassim, \& Erickson 2002). Depending on where LOFAR is sited, it could fill a critical frequency gap between the ionospheric cutoff near $20 \mathrm{MHz}$ and the frequency range of FASR $(\sim 0.1-30 \mathrm{GHz})$, corresponding to the outer corona. LOFAR is expected to make important contributions to our understanding of flares, CMEs and associated phenomena (e.g., type II radio bursts), and the interplanetary medium. Further afield, LOFAR's access to low frequencies will make it sensitive to Jovian-like emissions from magnetized exoplanets (e.g., Bastian, Dulk, \& Leblanc 2001; Zarka et al. 2003).

\section{Summary}

Radio observations of the Sun and solar-like stars continue to offer insights into the nature and range of magnetic activity. Recent progress on the radiophysics of the Sun has been in the areas of magnetic energy release in flares, understanding joint radio and X-ray observations of flaring loops, and in exploiting radio imaging of coronal mass ejections.

Stellar observations have benefited from the availability of a homogeneous array for VLBI imaging experiments, the VLBA. New limits have been placed on the nonthermal radio emission from both RS CVn binaries and classical flare stars. The upgrade of the Arecibo telescope now allows dynamic spectroscopy of radio bursts on stars to be performed over bandwidths an order of magnitude larger than was previously possible. A surprise has been the detection of radio emission from brown dwarfs, substellar objects at the bottom of the main sequence, which raises fascinating new questions about the magnetic dynamos and the nature of magnetic activity.

Indications are that for the solar-like stars and stellar systems, there is a convergence in our understanding of their radio emission and our understanding of solar radio emission. While stellar systems are extreme, even exotic physical environments, they are not so different that the lessons learned from the Sun cannot be applied.

A common theme for each of these developments described above has been the availability of new or upgraded instrumentation. With the next generation of powerful new radio instruments in the planning, design, or construction stage, progress on the radiophysics of the Sun and stars will only accelerate in future years. 
Acknowledgments. The National Radio Astronomy Observatory is a facility of the National Science Foundation operated under cooperative agreement by Associated Universities, Inc. I thank S. White for the radio HR diagram used in Fig. 1.

\section{References}

Abada-Simon, M., Lecacheux, A., Louarn, P. Dulk, G. A., et al. 1994, A\&A, 288,219

Abada-Simon, M., \& Aubier, M. 1997, A\&AS, 125, 511

Aschwanden, M. J., Wiehl, H. J., Benz, A. O., Kane, S. R. 1985, Solar Phys., 97,159

Aschwanden, M. J., Benz, A. O., Dennis, B. R., \& Schwartz, R. A. 1995a, ApJ, 455,347

Aschwanden, M. J., Montello, M. L, Dennis, B. R., \& Benz, A. O. 1995b, ApJ, 440,394

Basri, G. 2000, ARA\&A, 38, 485

Basri, G. 2004, in Stars as Suns, IAU Symp. 219, ed. A. K. Dupree \& A. O. Benz

Bastian, T. S. 2002, Innovative Telescopes and Instrumentation for Solar Astrophysics, Proc. SPIE v. 4853, eds. S. L. Keil \& S. V. Avakyan, SPIE: Bellingham, p. 98

Bastian, T. S. 1996, in Radio Emission from the Sun and Stars, ed. A. R. Taylor \& J. M. Paredes, ASP Conf. Ser. 93, ASP: San Francisco, p. 447

Bastian, T. S., \& Bookbinder 1987, Nature, 326, 678

Bastian, T. S., Bookbinder, J., Dulk, G. A., \& Davis, M. 1990, ApJ, 353, 265

Bastian, T. S., \& Gary, D. E. 1997, J. Geophys. Res., 102, 14031

Bastian, T. S., Benz, A. O., \& Gary, D. E. 1998, ARA\&A, 36, 131

Bastian, T. S., Maia, D., Pick, M. \& Kerdraon, A. 2001, ApJ, 558, L65

Bastian, T. S., Dulk, G. A., \& Leblanc, Y. 2000, ApJ, 545, 1058

Beasley, A. J., \& Güdel, M. 2000, ApJ, 529, 961

Benz, A. O., Perrenoud, M. R., Fürst, E., \& Hirth, W. 1981, Nature, 291, 210

Benz, A. O., Alef, W., \& Güdel, M. 1995, A\&A, 298, 187

Benz, A. O., Conway, J., \& Güdel, M. 1998, A\&A, 331, 596

Berger, E., Ball, S., Becker, K. M., Clarke, M., et al. 2001, Nature, 410, 339

Berger, E. 2002, ApJ, 572, 503

Byrne, P. B., Lanzafame, A. C., Sarro, L. M., \& Ryans, R. 1994, MNRAS, 270, 427

Cane, H. V., \& Reames, D. V. 1988, ApJ, 325, 895

Classen, H. T., \& Aurass, H. 2002, A\&A, 384, 1098

Cliver, E. W., Webb, D. F., \& Howard, R. A. 1999, Solar Phys., 187, 89

Dennis, B. R., Benz, A. O., Ranieri, M., \& Simnett, G. M. 1984, Solar Phys., 90,383 
Elgaroy, O. 1980, A\&A, 82, 308

Fekel, F. C., Moffet, T. J., \& Henry, G. W. 1986, ApJS, 60, 551

Fleishman, G. D., \& Kahler, S. W. 1992, ApJ, 394, 688

Fleishman, G. D., \& Melnikov, V. F. 1999, Proc. 9th European Meeting on Solar Physics, Magnetic Fields Solar Processes, Florence, Italy, ESA SP-448, p. 1247

Franciosini, E., \& Chiuderi Drago, F. 1995, A\&A, 297, 535

Gary, D. E., \& Keller, C. 2004, in press

Gopalswamy, N., \& Kundu, M. R. 1993, Solar Phys. 143, 327

Gopalswamy, N. Kundu, M. R., Manoharan, P. K. Raoult, A., Nitta, N., Zarka, P. 1997, ApJ, 486, 1036

Güdel, M. 1994, ApJS, 90, 743

Güdel, M. 2002, ARA\&A, 40, 217

Güdel, M., \& Benz, A. O. 1994, ApJ, 405, L63

Hall, D. S. 1976, IAU Colloq. 29, Multiple Periodic Variable Stars, ed. W. S. Fitch (Dordrecht: Reidel), p. 287

Hanaoka, Y. 1996, Solar Phys., 165, 275

Hanaoka, Y. 1997, Solar Phys., 173, 319

Isliker, H., \& Benz, A. O. 1994, A\&AS, 104, 145

Kassim, N. E., Perley, R. A., Erickson, W. C., \& Dwarakananth, K. S. 1993, AJ, 106, 2218

Kassim, N. E., Lazio, T. J. W., Erickson, W. C., Crane, P. C., Perley, R. A., \& Hicks, B. 2002, in The Universe at Low Radio Frequencies, Proc. IAU Symp. 199, ed. A. P. Rao, G. Swarup, \& G. Krishna, p. 474

Kathiravan, C., Ramesh, R., \& Subramanian, K. R. 2002, ApJ, 567, 93

Lecacheux, A., Rosolen, C., Davis, M., Bookbinder, J., et al. 1993, A\&A, 275, 670

Melrose, D. B., \& Dulk, G. A. 1982, ApJ, 259, 844

Mohanty, S., \& Basri, G. 2003, ApJ, 583, 451

Mutel, R. L., Lestrade, J. F., Preston, R. A., \& Phillips, R. B. 1985, ApJ, 289, 262

Mutel, R. L., Morris, D. H., Doiron, D. J., \& Lestrade, J. F. 1987, AJ, 93, 1220

Nakajima, T., Oppenheimer, B. R., Kulkarni, S. R., Golimowski, D. A., et al. 1995, Nature, 378, 463

Nishio, M., Yaji, K., Kosugi, T., Nakajima, H., \& Sakurai, T. 1997, ApJ, 489, 976

Pestalozzi, M. R., Benz, A. O., Conway, J. E., \& Güdel, M. 2000, A\&A, 353, 569

Ramesh, R., Kathiravan, C., \& Sastry, Ch. V. 2003, ApJ, 591, 163

Ransom, R. R., Bartel, N., Bietenholz, M. F., Leback, D. E., et al. 2003, ApJ, 572,487

Rutledge, R. E., Basri, G., Martín, E. L., \& Bildsten, L. 2000, ApJ, 538, L141

Seaquist, E. 1993, Rep. Prog. Physics., 56(9), 1145 
Stepanov, A. V., Kliem, B., Zaitsev, V. V., Fürst, E., et al. 2001, A\&A, 374, 1072

Strassmeier, K. G., Hall, D. S., Fekel, F. C., \& Sheck, M. 1993, A\&AS, 100, 173

Trigilio, C., Buerni, C. S., Umana, G., Rodonò, M., et al. 2001, A\&A, 373, 181

Uchida, Y. 1974, Solar Phys., 39, 431

van den Oord, G. H. J. 1996, in Radio Emission from the Sun and Stars, ed. A. R. Taylor \& J. M. Paredes, ASP Conf. Ser. 93, ASP: San Francisco, p. 263

Wagner, W. J., \& MacQueen, R. M. 1983, A\&A, 20, 136

White, S. M. 2000, in Radio Interferometry: The Saga and the Science, ed. D. G. Finley \& W. M. Goss, NRAO Workshop 27, p. 86

White, S. M., \& Kundu, M. R. 1997, Solar Phys., 174, 31

White, S. M., \& Franciosini, E. 1995, ApJ, 444, 342

White, S. M., Kassim, N. E., Erickson, W. C. 2002, in Innovative Telescopes and Instrumentation for Solar Astrophysics, Proc. SPIE v. 4853, ed. S. L. Keil \& S. V. Avakyan, SPIE: Bellingham, p. 111

Zarka, P., Treumann, R. A., Ryabov, B. P., \& Ryabov, V. B. 2001, APSS, 277, 293 

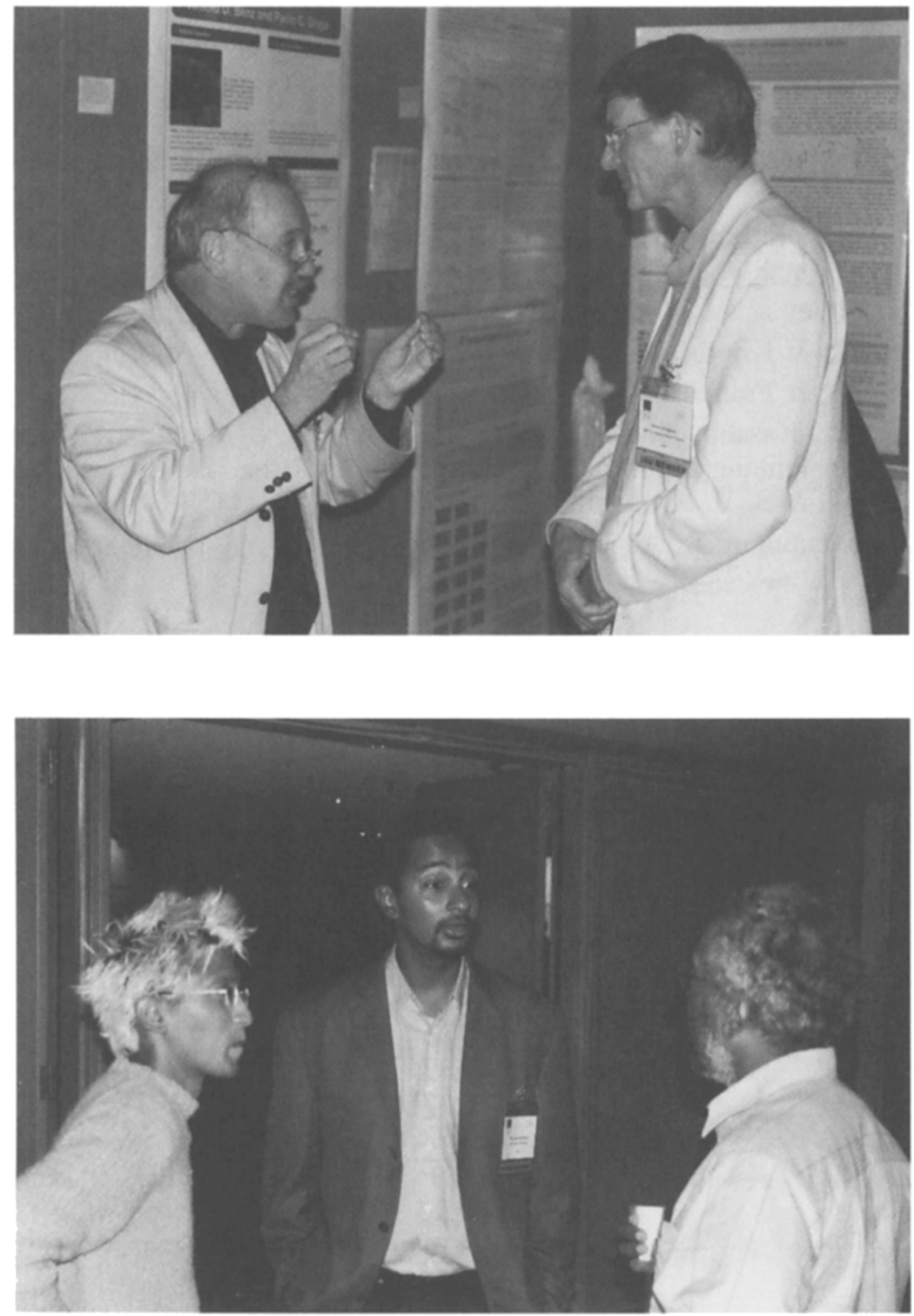